\title{
COVID-19 y educación ¿Cuál es el mal que afecta a Paraguay en el marco de América Latina?
}

\section{COVID-19 and education. Which is the evil that affects Paraguay in the context of Latin America?}

\author{
Nora Gluz ${ }^{a}$, Rodolfo Elías ${ }^{b}$
}

\begin{abstract}
Resumen
El artículo analiza las respuestas de los sistemas educativos latinoamericanos frente a la pandemia, poniendo atención especial al caso paraguayo. La pandemia llega a la región, en el marco de un "giro a la derecha" de gran parte de los gobiernos. Estos proyectos se oponen a los avances logrados en el derecho a la educación durante los gobiernos progresistas.

$\mathrm{Al}$ examinar las medidas asumidas durante la pandemia en distintos países de la región se identifican importantes tensiones: a) Los países cuyos gobiernos sostienen orientaciones mercantilistas tendieron a suspender las clases "bajo presión" de las comunidades priorizando el lucro sobre el resguardo de la vida en común; b) Las profundas desigualdades de género en países en que el patriarcado se evidenciaron aún más durante la pandemia, en que las fronteras entre escuela y hogar se diluyen, en especial en la docencia, donde la feminización del trabajo y desigualdades de género se potencian; c) En tercer lugar, las condiciones materiales en los hogares afectan la capacidad de respuesta y de trabajo a distancia. En el Paraguay, se puso al descubierto la debilidad del Ministerio de Educación (MEC) para generar y conducir una respuesta satisfactoria ante la crisis generada por el COVID-19.
\end{abstract}

Palabras clave: política educativa, COVID-19, América Latina, Paraguay.

\begin{abstract}
The article analyzes the responses of Latin American educational systems to the pandemic, paying special attention to the Paraguayan case. The pandemic reaches the region, within the framework of a "turn to the right" by a large part of the governments. These projects oppose the advances made in the right to education during progressive governments.

When examining the measures taken during the pandemic in different countries of the region, important tensions are identified: a) The countries whose governments hold mercantilist orientations
\end{abstract}

a Universidad de Buenos Aires, Argentina. b Universidad Nacional de Asunción, Facultad de Ciencias Sociales, Paraguay.

Correspondencia a: ngluz@campus.edu.ar

Recibido:

21 de mayo de 2020

Aceptado:

22 de junio de 2020

Artículo publicado en acceso abierto bajo la Licencia Creative Commons.

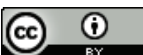

Cita:

Gluz, N. \& Elías. R. (2020).

COVID-19 y educación ¿Cuál es el mal que afecta a Paraguay en el marco de América Latina? Kera Yvoty: reflexiones sobre la cuestión social, 5 (número especial), $57-63$. 
tended to suspend the classes "under pressure" from the communities, prioritizing profit over the protection of life in common; b) The deep gender inequalities in countries where patriarchy was even more evident during the pandemic, in which the boundaries between school and home are blurred, especially in teaching, where the feminization of work and gender inequalities are enhanced; c) Thirdly, the material conditions in the homes affect the capacity to respond and to work at a distance. In Paraguay, the weakness of the Ministry of Education (MEC) to generate and conduct a satisfactory response to the crisis generated by COVID-19 was exposed.

Keywords: education policy, COVID-19, Latin America, Paraguay.

\section{Introducción: o ¿cómo llega la pandemia a América Latina?}

La concentración extraordinaria de la riqueza global en una elite, acelerada a partir de 2010, así como las "democracias capturadas" (OXFAM, 2018) afectan sensiblemente a América Latina, que continúa siendo la región más desigual del mundo a pesar de las políticas públicas de redistribución y reconocimiento social implementadas por los gobiernos progresistas en los albores del siglo XXI.

La pandemia llega a la región, en el marco del "giro a la derecha" de gran parte de los gobiernos, y del despliegue de políticas educativas mercantilizadoras. Estos proyectos se oponen a los importantes avances en la materialización del derecho a la educación durante los gobiernos progresistas en América Latina, aunque también en dicho período persistieron tendencias neoliberales en algunos países (Saforcada \& Vassiliades, 2011; Feldfeber \& Gluz, 2020).

Las políticas globales hacia la escolarización promovieron desde los '9o diferentes formas de privatización, así como el desarrollo de sistemas estandarizados de evaluación con énfasis en estándares educativos, que se reforzó con la inclusión de la tecnología en educación y administración (Verger et al., 2017). La mercantilización se intensificó a partir del ascenso reciente de gobiernos liderados por fuerzas sociales de derecha en alianzas con corrientes pentecostalistas del campo de la teología de la prosperidad (creada en los Estados Unidos), alineados contra la agenda del movimiento feminista con un discurso de defensa de los valores de la familia tradicional (Semán, 2019), y con sectores empresariales a través de formatos de institucionalización capitalista. Ello dio lugar a a nuevas alianzas públicoprivadas en educación que operan a través de mecanismos de terciarización de funciones estatales; se fundamentan en el management y sus nuevas expresiones vinculadas al emprendedurismo; así como en la difusión de nuevas explicaciones individualizantes que bajo el velo de la educación emocional, el liderazgo educativo y el couching provocan la desvalorización del trabajo pedagógico del profesorado (Cornejo et al. 2018; Martinis \& Falkin, 2017; Croso, 2016; Feldfeber \& Oliveira, 2016).

Ello ha afectado centralmente al cuerpo docente, iniciando $\mathrm{y} / \mathrm{o}$ dando continuidad a procesos de deslegitimación de la docencia y de las organizaciones de educadores (Burns \& Luque, 2014), a través del desmantelamiento de los sistemas de formación docente a cargo del Estado, contratando profesores que se formaron en otras disciplinas a los que se les promociona con certificaciones alternativas, entre otras estrategias. Programas como Teach for América nacidos en EEUU y que luego se ha puesto en marcha en nuestros países con el nombre de Teach for All expresan esa lógica.

En el marco de este deterioro, las propuestas de "seguir aprendiendo" a distancia, fue la demanda hacia ese colectivo profesoral hasta entonces tan criticado, al iniciarse el aislamiento social ante la pandemia. 


\section{Medidas comunes en escenarios desiguales $^{1}$}

En el mes de marzo todos los sistemas escolares interrumpieron sus clases ante las medidas de aislamiento social adoptada por los gobiernos; dando paso a una rápida y exigida transformación de los modos de transmisión de modo de sostener en el hogar, algunas dimensiones de los procesos de transmisión propios de la escuela. A través de plataformas digitales o de la $\mathrm{TV}$, radios públicas y la distribución de cuadernillos y ejercitarios para sortear las profundas desigualdades de acceso a los medios digitales, el sistema educativo mantuvo su "presencia". Pronto quedó al descubierto que la escuela en el capitalismo contemporáneo es más que ello, y se avanzó en medidas sociales como apoyos alimentarios y/o económicos a las familias, de alcance ciertamente limitado frente a la gravedad de la falta de ingresos en los hogares cuyos miembros trabajan en el mercado informal. Se trató junto a la extensión de las barriadas populares, falta de servicios esenciales, de una realidad propia de nuestra región donde los antecedentes de lo ocurrido en los países centrales no ofrecieron experiencias de las cuales aprender y por ende contribuir a anticipar medidas. Si bien las intervenciones recién descriptas parecieran marcar caminos comunes, en su relación las desigualdades nacionales y las orientaciones políticas de los gobiernos se marcan las diferencias.

Un análisis somero de las principales medidas en distintos países de la región da cuenta de al menos dos importantes tensiones. En primer lugar, los países

1 Este apartado fue desarrollado en base a los aportes de integrantes del GT CLACSO Políticas Educativas y Derecho a la Educación: Pablo Imen, Ingrid Sverdlick, Rosario Austral y Fernanda Saforcada (Argentina), Aurora Santiago (Puerto Rico), Carmen Núñez, Jenny Assael y Vicente Sisto (Chile), Gabriela Walder (Paraguay), Carmen Rodríguez (España), Cibele Rodríguez y Heleno Araujo (Brasil), Ricardo Cuenca (Perú), Carmen Caamaño (Costa Rica), Georgina Gutierrez (México), Eloisa Bordoli (Uruguay), Maikel Pons (Cuba), Dayana Cardona y Felipe Mora (Colombia), David Añazco (Ecuador). cuyos gobiernos sostienen orientaciones mercantilistas tendieron a suspender las clases "bajo presión" de las comunidades tal es el caso de Chile y Brasil- priorizando el lucro o una acepción de él por sobre el resguardo de la vida en común. Ello se expresa en la preeminencia de un discurso eficientista en el que se proclama no detener la economía y seguir aprendiendo, aunque ello desconozca las brechas de desigualdad que obturan las condiciones para que ese aprender tenga lugar. Países como Argentina contrariamente, han acompañado la suspensión de clases con inmediatas medidas destinadas a atenuar las desigualdades como la exigencia a empresas de telefonía móvil o internet el acceso gratuito a plataformas educativas institucionales, distribución de netbooks y producción en tiempo record de programas educativos y cuadernillos para garantizar la accesibilidad, medida también asumida por México.

Los discursos eficientistas se articulan con discursos meritocráticos que se expresan en una presión sobre lo que miden como "esfuerzo" estudiantiles y que individualizan las consecuencias sociales de la pandemia. Ejemplos paradigmáticos como Chile, que cuenta con un sistema escolar sostenido a través del financiamiento por la demanda y cuya preocupación es medir la "asistencia" escolar en estas nuevas coordenadas, como si el cumplimiento con los requerimientos escolares pudiera resolverse a voluntad. Desde otras bases educativas, Paraguay ha intentado condicionar la alimentación a la entrega de trabajos prácticos desconociendo la emergencia social en los hogares y exigiendo contraprestaciones para garantizar derechos.

Un segundo eje de tensiones deviene de las profundas desigualdades de género en países en que el patriarcado está arraigado culturalmente. Las desigualdades de género son un potente-aunque invisible- vector de desigualdad que expresa consecuencias en una profesión altamente feminizada como la docencia. En condiciones donde las 
fronteras entre escuela y hogar se diluyen, y en sociedadesen lasqueel trabajodecuidado no remunerado se distribuye de forma desigual entre las familias u organizaciones de la sociedad civil, el Estado y el mercado; el aislamiento social devuelve a las familias esta función. En el caso de la docencia, feminización del trabajo y desigualdades de género se potencian, combinándose la intensificación del trabajo docente frente a la adecuación de tareas para el trabajo a distancia, el acompañamiento pedagógico a sus propios hijos e hijas y la sobrecarga de tareas domésticas. Es de destacar que en algunos países como Paraguay y Argentina se ha reportado un aumento de la violencia de género en los hogares y un aumento de los feminicidios evidenciando las dificultades de esa continuidad pedagógica en esos hogares, frente a los que la escuela en su dimensión física representaba un ámbito de protección y cuidado en sentido amplio. Cuba parece ser el único país que ha sostenido la presencialidad opcional en los jardines de infantes para garantizar las tareas de cuidado.

En tercer lugar, las condiciones materiales en los hogares que afectan la capacidad de respuesta y de trabajo a distancia. Desde factores básicos como acceso a la alimentación y a la salud, así como la infraestructura de los hogares son condicionantes de las posibilidades concretas de organización del trabajo escolar de forma remota. Si el acceso a internet y la posesión de medios digitales ha sido muy visibilizada, más ocultas han quedado las condiciones de hacinamiento y el acceso a servicios básicos como plafón material para la conexión física y actitudinal el estudio. En países donde el giro a la derecha en años previos había debilitado la acción pública, los ministerios tienen mayores dificultades para remontar los fuertes recortes en sus políticas sociales, en especial, en salud y educación. En muchos casos ello se agudiza con la falta de diálogo de los ministerios de educación con las organizaciones de educadores, estudiantes, madres y padres que deriva en medidas unilaterales inadecuadas a la diversidad de situaciones como las de escuelas rurales, en comunidades indígenas, en barrios populares.

\section{La centralidad de lo público ¿con plataformas privadas? Los desafíos frente a la privatización y mercantilización}

Es indudable que sostener desde la escuela la educación que se desarrolla en los hogares depende en parte de las condiciones de conectividad de los hogares. En países que venían de gobiernos progresistas como Uruguay, gracias a la alta conectividad extendida por todo el país, el Plan Ceibal, especialmente, la primaria pudo continuar con actividades virtuales a través de las plataformas educativas, pero también del vínculo de las escuelas con sus contextos, donde la experiencia del Programa Maestro Comunitario generó un plafón para vehicular el contacto con las familias que no cuentan con internet.

Pero si bien estas son las condiciones de posibilidad, no son suficientes. Se ha otorgado una centralidad de las plataformas digitales como solución a los problemas educativos desconociendo su impacto en la mercantilización de la escolarización. En palabras de Van Dijck (2016):

No hay un solo nicho de la socialidad online que sea puramente público o sin fines de lucro, por el sencillo motivo de que es difícil que algo pueda prosperar si no cuenta con el apoyo de la infraestructura que Google, Facebook, Twitter y otras compañías "han hecho social. (p. 156)

El avance de las plataformas privadas y de los softwares educativos y, a través de éstos, la injerencia de los fondos privados en las escuelas públicas entrega un mercado cautivo a empresas multinacionales. Como se evidencia ya en Estados Unidos, escuelas con fondos insuficientes aceptan acuerdos con empresas como Google y Facebook que les ofrece instructores y recursos para utilizar sus desarrollos tecnológicos; a la vez 
que dan acceso a un extraordinario cúmulo de información sobre los estudiantes para futuros negocios. Sin duda negocios y pérdida de soberanía pedagógica se anudan en estos nuevos modos de mercantilización.

Esto se intensificaría frente al triunfo de los modelos de Home Schooling (escuelas en el hogar), propuesta que hoy impulsan las nuevas derechas. En Ecuador, por ejemplo, y en el marco de un recorte presupuestario, algunos funcionarios han indicado que existiendo esta alternativa no sería necesario construir más escuelas. Desfinanciamiento y privatización construyen allí un matrimonio perfecto.

\section{Paraguay en el escenario regional: lecciones, tensiones y rumbos perdidos}

El sistema educativo paraguayo enfrenta una marcada desigualdad ${ }^{2}$ desde hace décadas y los últimos gobiernos profundizaron esta situación en el campo escolar. En 2018, un año antes de la expansión del COVID-19, el gasto público en educación como porcentaje del PIB fue de 3,6\%, un marcado descenso frente al $5,3 \%$ invertido hace dos décadas (2000) y del $5,1 \%$ hace una (2010) (MEC, 2019). Ello en un contexto de déficit en la infraestructura escolar, escaso apoyoa la formacióny trabajo docente, estancamiento de la cobertura, altas tasas de abandono, en especial en la educación secundaria, escasa atención a la diversidad social, cultural y lingüística en los programas educativos y un avance de visiones conservadoras en contra de la perspectiva de derecho y el enfoque de género ${ }^{3}$.

En este escenario se produce la

2 Según datos de la EPH, alrededor del $20 \%$ de la población vive en condiciones de pobreza y el $4 \%$ en pobreza extrema, situación que afecta en mayor medidas a niñas, niños y adolescentes y la población rural, donde la pobreza rural infantil afecta a más del 40\% de la población entre o y 17 años (DGEEC, 2019). 3 Para más información, ver: Perfiles de exclusión educativa en Paraguay (UNICEF, 2016), Informe Beining +20 (Elías, 2015), Insumos escolares básicos en Paraguay (World Bank, 2013), Mapa Escolar (MEC, 2020) y Observatorio Educativo Ciudadano (2020). pandemia. El Ministerio de Salud tomó medidas rápidas y hasta la fecha, los contagios y fallecimientos se mantienen controlados. Sin embargo, en el campo educativo, la pandemia puso al descubierto la debilidad del Ministerio de Educación y Ciencias (MEC) para generar y conducir una respuesta satisfactoria ante la crisis generada por el COVID-19.

El MEC, al igual que otros países de la región, canceló las clases presenciales y pasó a desarrollar una estrategia de educación a distancia utilizando una plataforma denominada "Tu escuela en casa". ${ }^{4}$ Estas medidas y otras que siguieron, generaron una serie de críticas y descontentos de la ciudadanía. Las organizaciones de docentes y estudiantes exigieron la conformación de una mesa para discutir y definir un plan de educación en el contexto de la pandemia que constituya una respuesta adecuada a las necesidades de la población y a las condiciones del país. Lejos de esta demanda, el ministro respondió condicionando la entrega de los kit de alimentos a cambio del cumplimiento de las tareas escolares (Unima Hora, 2020). lo que demostró la poca comprensión de la situación económica, social y educativa en la que se encuentra un amplio sector de la población, que no tiene las condiciones materiales ni culturales para realizar un acompañamiento escolar de sus hijos e hijas.

Las críticas y denuncias realizadas llevaron a la interpelación del Ministro de Educación en el Congreso Nacional y recién frente a la movilización y presión ciudadana, el MEC presentó un plan de acción y convocó a una mesa de diálogo. Las principales organizaciones de docentes, estudiantes y madres $y$ padres han condicionado dicha participación a una definición clara en cuanto a la integración

\footnotetext{
4 Esta plataforma se crea a partir de un acuerdo entre el MEC y MICROSOFT, que, según el Ministro, "cede gratuitamente la plataforma para poner en marcha "Tu escuela en casa" que tendrá capacidad para unos 1.200.00o usuarios".
} 
de la mesa y al poder de decisión que pueda tener este espacio.

\section{Pandemia con desigualdad, ese es el "mal"}

La rápida expansión del COVID-19 $y$ el recurso a acciones excepcionales por parte de los Estados, trastocó los patrones de convivencia social, los lazos comunitarios y cerró fronteras internas y externas posicionando al "otro" como potencial peligro.

Entre las drásticas medidas asumidas, la suspensión a escala prácticamente mundial de las clases presenciales alteró no sólo los mecanismos de transmisión cultural consagrados, sino las estrategias de cuidado y construcción de infancias y adolescencias. La excepcionalidad de la situación como patrón común, dejó poco margen para la anticipación a los gobiernos de todo el planeta, pero sin lugar a dudas, afectó de modo diferencial allí donde las desigualdades son la regla y donde la debilidad de los recursos y capacidades estatales restringen los márgenes de actuación.

La situación de nuestra región y de Paraguay en particular, deja en claro que lo que, como expresa Therborn (2017); la desigualdad afecta "el bienestar corporal (la igualdad vital) y la autonomía personal (la igualdad existencial, en particular en lo que respecta a las estructuras etnorracistas y familiares de género)". La pandemia interpone a esas desigualdades preexistentes nuevos riesgos, pero que operan sobre bases a las que esperamos pongan tanto o más en cuestión que la salud de la población. Los gobiernos que fortalecen la igualdad de derechos marcan la diferencia.

\section{Referencias}

Burns, B., \& Luque, J. (2014). Profesores excelentes: Cómo mejorar el aprendizaje en América Latina y el Caribe. Banco Mundial.

Cornejo, R., Assaél, J., Redondo, J., \& Insunza. J. (2019). Public Education and Student Movements: The Chilean Rebellion under a Neoliberal Experiment. British Journal of Sociology of Education, 4o(4).

Croso, C., \& Magalhães, G. (2016) Privatização da educação na américa latina e no caribe: tendências e riscos para os sistemas públicos de ensino. Educação E Sociedade, (37).

Diario Hoy. (19 de marzo 2020). MEC lanza plataforma para que alumnos tengan clases virtuales durante pausa por COVID-19. https://www.hoy.com.py/ nacionales/mec-lanza-plataformapara-que-alumnos-tengan-clasesvirtuales-durante-pausa-por-covid-19

Dirección General de Estadística, Encuestas y Censos, DGEEC. (2019). Encuesta Permanente de Hogares (EPH). DGEEC.

Elias, R. (2015). Educación y capacitación de la mujer: El sistema educativo paraguayo entre la expansión de la demanda social y la arremetida conservadora. En: L. Bareiro, C. Soto (Eds.), Paraguay a 20 años de Beijing 1995. CDE.

Feldfeber, M. \& Oliveira, D. (2016). Políticas Educativas en América Latina en el siglo XXI. Balance y perspectivas. Revista del Instituto de Investigaciones en Ciencias de la Educación, 1.

Feldfeber, M., \& Gluz, N. (2020). Las políticas educativas a partir del cambio de siglo: alcances y límites en la ampliación del derecho a la educación en Argentina. Revista Estado y Políticas Públicas, (13).

Martinis, P., \& Falkin, C. (2017). Aspectos pedagógicos y de política educativa involucrados en los procesos de universalización del derecho a la educación. En A. Cirstóforo, P. Martinis, M. Míguez, N. Viscardi (Eds.), Derecho a la educación y mandato de obligatoriedad en la enseñanza media. UdelaR.

Ministerio de Educación y Ciencia, MEC (2020). Mapa Escolar. https:// mapaescolar.mec.gov.py/mapa escolar/ 
Observatorio Educativo Ciudadano. (2020). La educación de niños, niñas y adolescentes en cuarentena. https:// observatorio.org.py/

OXFAM. (2018). Democracias capturadas: el gobierno de unos pocos. CLACSO.

Saforcada, F., \& Vassiliades, A. (2011). Las leyes de educación en los comienzos del siglo XXI: del neoliberalismo al postconsenso de Washington en América del Sur. Revista Educação $\mathcal{E}$ Sociedade, $32(115)$.

Semán, P. (2019); ¿Quiénes son? ¿Por qué crecen? ¿Enquécreen? Pentecostalismo y política en América Latina. Revista Nueva Sociedad, (280).

Therborn, G. (2017). La dinámica de la desigualdad.: New Left Review, (103), 69-90. https://rebelion.org/ docs/228983.pdf

Ultima Hora. (27 de abril de 2020). Petta condiciona entrega de kits alimenticios a cambio de tareas. https://www. ultimahora.com/petta-condicionaentrega-kits-alimenticios-cambiotareas-n2882299.html
UNICEF. (2016). Perfiles de la exclusión educativa en la República de Paraguay. https://www.unicef.org/ paraguay/informes/perfiles-de-laexclusi $\% \mathrm{C}_{3} \% \mathrm{~B}_{3}$-educativa-en-larep $\% \mathrm{C}_{3} \% \mathrm{BAblica-de-paraguay}$

Van Dicjk, J. (2016). La cultura de la conectividad: una historia crítica de las redes sociales. Siglo XXI.

Verger, A., Moschetti, M., \& Fontdevilla, C. (2017). La privatización educativa en América Latina: Una cartografía de políticas, tendencias y trayectorias. Internacional de Educación.

World Bank. (2013). Insumos escolares básicos en Paraguay. https://es.scribd. com/document/408080766/InsumosEscolares-Basicos-en-Paraguay-BMpdf

\section{Sobre los Autores}

Nora Gluz

Docente investigadora UNGS -UBA. Cocoordinadora GT CLACSO Políticas Educativas y Derecho a la Educación.

Rodolfo Elías

Docente de FACSO, Coordinador académico de FLACSO/Paraguay. Cocoordinador GT CLACSO Políticas Educativas y Derecho a la Educación. 